\title{
Bone morphogenetic protein-12 inducing tenogenic differentiation of mesenchymal stem cells enhances healing of linea alba incision
}

\author{
DONG WANG, ZHEN-LING JI, JING-MIN WANG and YU-YAN TAN \\ Department of General Surgery, Zhongda Hospital, School of Medicine, \\ Southeast University, Nanjing, Jiangsu 210009, P.R. China
}

Received March 4, 2018; Accepted October 8, 2018

DOI: $10.3892 /$ etm.2018.6861

\begin{abstract}
The objective of this study was to investigate the curative effects of mesenchymal stem cells' tenogenic differentiation on linea alba incision healing induced by bone morphogenetic protein-12. Mesenchymal stem cells were isolated and induced by $10 \mathrm{ng} / \mathrm{ml}$ of bone morphogenetic protein-12 for $48 \mathrm{~h}$. Expression of scleraxis, collagen I and collagen III were examined at $48 \mathrm{~h}, 5$ and 7 days to investigate the tenogenic differentiation. The expression of scleraxis increases continually even in the absence of bone morphogenetic protein-12 for 5 days $(\mathrm{P}<0.01)$. The expression of collagen I and III requires persistent inducing. Then fifty Sprague-Dawley rats were randomly divided into five groups: negative control, positive control, sham group, native mesenchymal stem cells and tenogenically differentiated mesenchymal stem cells. Tensiometric testing and modified semiquantitative histological analysis were performed to explore the curative effects. The tension levels in the positive control, sham, native mesenchymal stem cells and tenogenically differentiated mesenchymal stem cells were 44, 41.8, 51.6 and $69.7 \%$, respectively, compared with the negative control. Tenogenically differentiated mesenchymal stem cells exhibited a greater increase in tension compared with positive control, sham and native mesenchymal stem cell groups $(\mathrm{P}<0.05)$. From the sections stained with Masson's Trichrome, collagen organization and amount of tenogenically differentiated mesenchymal stem cells was better than the other three groups $(\mathrm{P}<0.05)$. In conclusion, mesenchymal stem cells' tenogenic differentiation induced by bone morphogenetic protein-12 can enhance linea alba incision healing.
\end{abstract}

Correspondence to: Dr Zhen-Ling Ji, Department of General Surgery, Zhongda Hospital, School of Medicine, Southeast University, 87 Ding jia qiao, Nanjing, Jiangsu 210009, P.R. China E-mail: zlji@vip.sina.com

Key words: linea alba incision, incisional hernia, mesenchymal stem cells, bone morphogenetic protein-12

\section{Introduction}

Incisional hernia is the most common complication following abdominal surgery. The reported incidence after midline laparotomy ranges from 3 to $26 \%$ and increases rapidly if the native operation is complicated by wound infection or other conditions $(1,2)$. With the development of materials and techniques, substantial attention is currently being paid to hernioplasty. From the perspective of prevention, more work should be performed before hernia occurs. The incision healing depends on the balance of collagen synthesis, remodeling and degradation (3). The linea alba consists of transverse and oblique collagen fibers $(4,5)$, and cutting off these fibril bundles constitutes the anatomical basis of midline incisional hernia. Collagen regeneration and remodeling, especially the mature form of type I collagen (6), is the most important event in the healing of the midline incision.

For collagen synthesis, mesenchymal stem cells (MSCs) are widely used as seed cells in tissue regeneration medicine because of their easy gaining, quick proliferation and multiple differentiating ability. There have been few studies focusing on MSCs for incision healing. Stoff et al (7) injected MSCs into intracutaneous incisions, which significantly inhibited scar formation and increased the tensile strength of the skin wounds. Heffner et al (8) combined platelet-rich plasma, collagen and MSCs to investigate the healing effect on midline laparotomy incisions. The results indicated a marked improvement in abdominal wall strength, and histological evaluation confirmed increased vascularity and collagen abundance.

Bone morphogenetic proteins (BMPs) belong to the family of transforming growth factor $\beta$, which can induce MSCs differentiating into different tissues. Unlike other BMPs, BMP-12 has been reported to be less osteo-chondrogenic and to induce tendon rather than bone formation in vivo, and has already made some achievements in the field of tendon injuries $(9,10)$. The objective of this study is to investigate the curative effects of MSCs' tenogenic differentiation and transplantation on incision healing induced by BMP-12. To our knowledge, this study is a novel exploration of MSCs' regenerative application in abdominal wall surgery. 


\section{Materials and methods}

Laboratory animals and groups. Fifty Sprague-Dawley rats (Silaike Inc., Shanghai, China), weighing between 150 and $200 \mathrm{~g}$, were randomly divided into five groups: negative control (A), positive control (B), sham group (C), native MSCs (D) and tenogenically differentiated MSCs (E). Ten rats were included in each group. All experiments were conducted with the approval of the Animal Care and Use Committee of Southeast University (Nanjing, China).

Group A did not undergo any operation and nothing was implanted. Group B only underwent operation, but did not implant anything. Group $\mathrm{C}$ undergo operation, but only sponge scaffold was implanted into the linea alba incision. Group D undergo operation and sponge scaffold with native MSCs were implanted. Group E undergo operation and sponge scaffold with tenogenically differentiated MSCs were implanted.

Isolation and culture of MSCs. The rat bone marrow-derived MSCs were prepared as described $(11,12)$. Briefly, bone marrow was collected by flushing the femur and tibia with medium, and MSCs were isolated by gradient centrifugation for $30 \mathrm{~min}$ at room temperature, using Ficoll-Paque PLUS $(1.077 \mathrm{~g} / \mathrm{ml}$; GE Healthcare Life Sciences, Little Chalfont, UK). After centrifugation, cell pellets were resuspended in Dulbecco's modified Eagle's medium with low glucose (Invitrogen; Thermo Fisher Scientific, Inc., Waltham, MA, USA), supplemented with $10 \%$ fetal bovine serum and incubated at $37^{\circ} \mathrm{C}$ in a humidified atmosphere containing $5 \% \mathrm{CO}_{2}$ with regular replenishment of medium every two to three days.

BMP-12 inducing tenogenic differentiation of MSCs. MSCs at passage 3 were plated at a density of $5 \times 10^{2}$ cells $/ 35 \mathrm{~mm}$ dish and treated with BMP-12 (Sigma-Aldrich; Merck KGaA, Darmstadt, Germany) of $10 \mathrm{ng} / \mathrm{ml}$ for $48 \mathrm{~h}$. Then, BMP-12 was removed and replaced by normal medium without BMP-12 for another 5 days. reverse transcription-quantitative polymerase chain reaction was used to evaluate the expression of scleraxis (SCX), collagen I and collagen III at $48 \mathrm{~h}, 5$ and 7 days. The gene-specific primers are presented in Table I. The relative quantity of target gene expression was analyzed using the comparative $\mathrm{C}_{\mathrm{T}}\left(2^{-\Delta \Delta \mathrm{Cq}}\right)$ method (13). GAPDH was used as an endogenous reference gene.

In vivo implantation. The MSCs-sponge complex was built as previously described (14). MSCs were suspended in growth medium at $1 \times 10^{6}$ cells $/ \mathrm{ml}$, and $0.5 \mathrm{ml}$ was seeded onto sterilized 2x10 mm collagen sponge scaffolds. Cell-seeded scaffolds were placed in culture dishes and incubated for $2 \mathrm{~h}$ in a minimum volume of normal medium (Fig. 1A), after which more medium was applied to submerge the scaffolds for $24 \mathrm{~h}$. Then, the MSCs-sponge complex was cultured for an additional 7 days using $10 \mathrm{ng} / \mathrm{ml}$ of BMP-12 or normal medium.

Animals were anesthetized with $0.3 \%$ pentobarbital sodium, and the abdominal skin was sterilized with iodophor. A longitudinal midline skin incision was made to expose the linea alba, and a $10 \mathrm{~mm}$ long defect was created along the linea alba (Fig. 1B), referring to a previous study (15). Then, the linea alba incision was closed using 5-0 prolene (Ethicon, Inc., Cincinnati, OH, USA) with or without the
MSCs-sponge complex (Fig. 1C). The suture/incision ratio was $4: 1$.

After the operation, the animals were raised for another 4 weeks and then euthanized. The whole abdominal wall was obtained for further analysis (Fig. 1D). The sample including the linea alba scar was pruned into two parts with widths of $5 \mathrm{~mm}$. One piece was preserved in saline for tensiometric testing, and the other was fixed in $10 \%$ neutral buffered zinc-formalin for histological analysis.

Tensiometric testing. Mechanical testing of the linea alba scar was performed within $6 \mathrm{~h}$ of tissue harvest. According to the previous study (16) and our pilot trial, the sample for tensiometric testing requires special pruning because the ventral muscles have a remarkably weaker anti-tensile capacity than the linea alba. As shown in Fig. 2A, only $2 \mathrm{~mm}$ of the midline scar was preserved compared to $5 \mathrm{~mm}$ of ventral muscles. It was loaded into the grips of an Instron tensiometric machine (Instron, Norwood, MA, USA) equipped with a $250 \mathrm{~N}$ load cell. Tissue samples preloaded with $1 \mathrm{~N}$ force were then loaded at a rate of $10 \mathrm{~mm} / \mathrm{min}$ (17) until complete failure (Fig. 2B). The load and displacement data recorded were used to determine the maximum force required for failure of the linea alba scar. Because of the notable deformation of the tissues, the modulus of elasticity and displacement index were not included in this study. Data analysis was conducted using Instron's Bluehill software package.

Histological analysis. The fixed tissues were cut transversely to present the healing of the linea alba scar and stained with Masson's Trichrome. The scale used in our study was a modified semiquantitative histological analysis (Table II), which is analogous to that described by Rice et al (17). Two blinded observers determined the collagen amount and organization. Staining depth of the collagen reflected the amount. Organization indicated as the orientation of fibers. Each example was examined at four random sites to get an average score.

Staining depth was evaluated using ImageJ (Version $1.50 \mathrm{~g}$, National Institutes of Health, Bethesda, MD, USA) after the graph was converted to black and white color. Group A was set as the control with an average grayscale of 170.0 (disorganized) indicated the average grayscale was below 120, 1 (mildly organized) indicated the average grayscale was between 121 and 130, 2 indicated the average grayscale was between 131 and 140, 3 (moderately organized) indicated the average grayscale was between 141 and 150, 4 indicated the average grayscale was between 151 and 160, and 5 (well organized) indicated the average grayscale was between 161 and 170 .

The four random sites' microphotographs of each sample with a magnification $\times 200$ was examined under light microscopy to assess the fiber orientation. The collagen of group A was set as the parallel fiber for quality control. If the fibers of another group and group A tended to be parallel, the angle between the two was small, otherwise the angle between the two increased (18,19). 0 (none) indicated the angle was below $50^{\circ}, 1$ (mild) indicated the angle was between $50^{\circ}$ and $59^{\circ}, 2$ indicated the angle was between $60^{\circ}$ and $69^{\circ}, 3$ (moderate) indicated the angle was between $70^{\circ}$ and $79^{\circ}, 4$ indicated the angle was between $80^{\circ}$ and $89^{\circ}$, and 5 (abundant) indicated the angle was between $90^{\circ}$ and $100^{\circ}$. 
Table I. Primers for RT-qPCR analysis.

\begin{tabular}{llc}
\hline Gene & Forward sequence & \multicolumn{1}{c}{ Reverse sequence } \\
\hline Scx & 5'-AGAGACGGCGGCGAGAAC-3' & 5'-AATCGCCGTCTTTCTGTCACG-3' \\
Col I & 5'-AATGGTGCTCCTGGTATTGC-3' & 5'-GGTTCACCACTGTTGCCTTT-3' \\
Col III & 5'-AGCTGGACCAAAAGGTTGATG-3' & 5'-GACCTCGTGCTCCAGTTAGC-3' \\
GAPDH & 5'-CCTGGCCAAGGTCATCCAT-3' & 5'-GTCATGAGCCCTTCCACGAT-3' \\
\hline
\end{tabular}

RT-qPCR, reverse transcription-quantitave polymerase chain reaction; Scx, scleraxis; Col, collagen.

Table II. Modified histological scoring system for microscopic evaluation.

\begin{tabular}{lllll}
\hline & & & Score & \\
\cline { 2 - 5 } Collagen & 0 & 1 & 3 & 5 \\
\hline $\begin{array}{llll}\text { Organization } \\
\text { Amount }\end{array}$ & Disorganized & Mildly organized & Moderately organized & Well organized \\
\hline
\end{tabular}

Staining depth of the collagen reflected the amount. Organization indicated as the orientation of fibers.
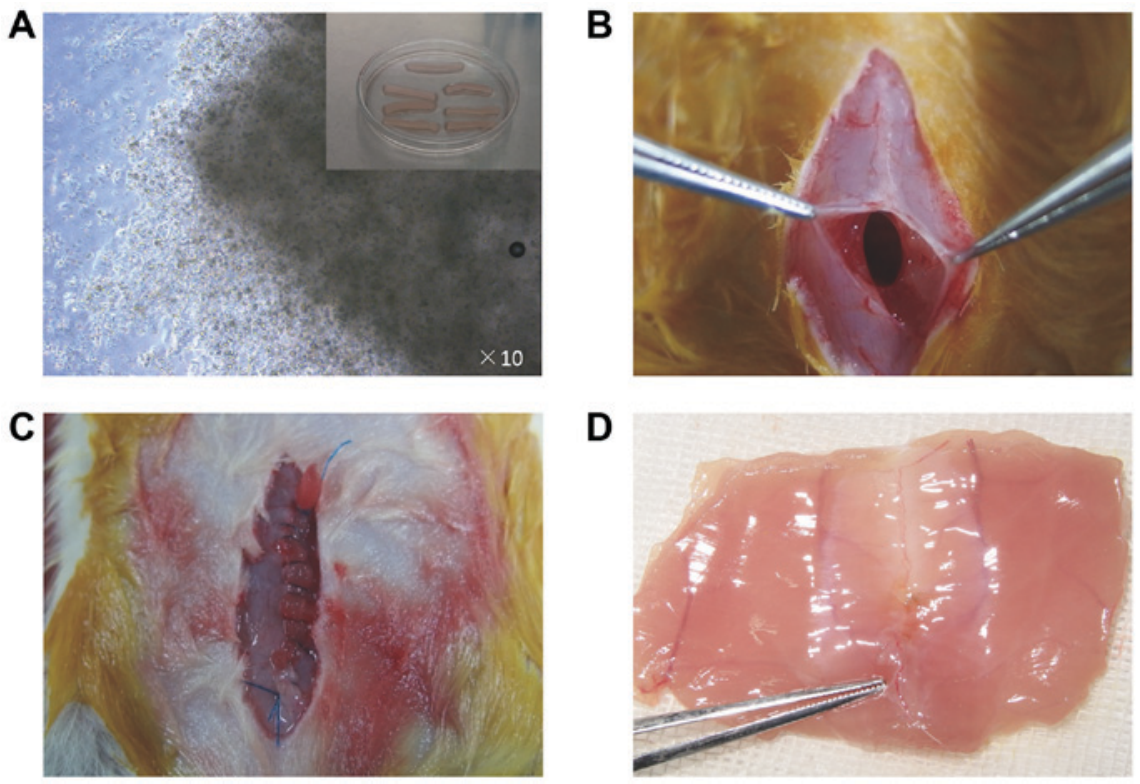

Figure 1. In vivo implantation. (A) Preparation of MSC-sponge complex with MSCs growing on the sponge; (B) Creation of linea alba incision; (C) Closure of the incision with MSC-sponge complex embedded into the incision; (D) Sample of healed linea alba incision with tenogenically differentiated MSCs. MSCs, mesenchymal stem cells.
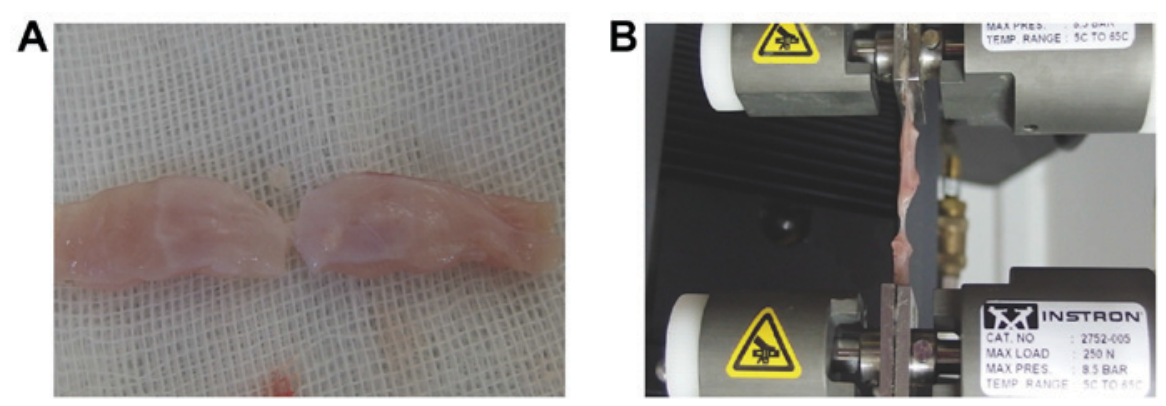

Figure 2. Tensiometric testing. (A) Special pruning of linea alba sample with $2 \mathrm{~mm}$ of the midline scar preserved; (B) Tensiometric test using Instron tensiometric machine. 

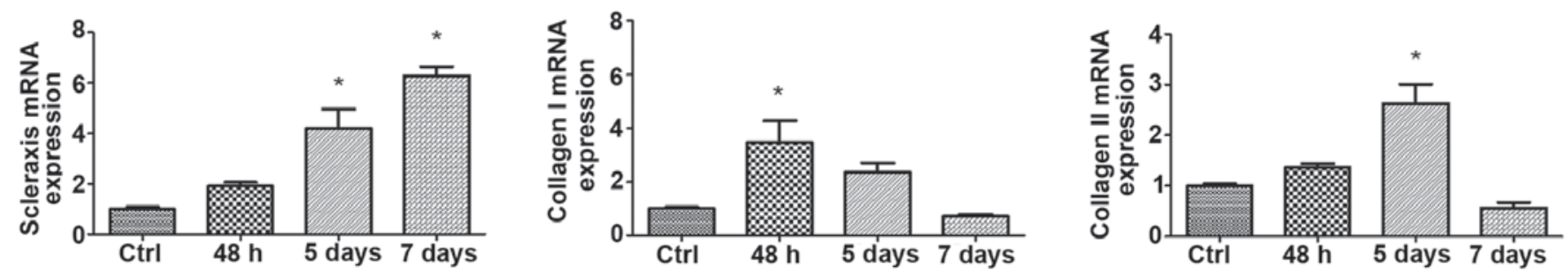

Figure 3. BMP-12 induces MSCs tenocyte differentiation. Increasing expression of SCX persists even if BMP-12 is removed. However, increasing expression of collagen I and collagen III require persistent induction by BMP-12. ${ }^{*} \mathrm{P}<0.05$. BMP, bone morphogenetic protein; MSCs, mesenchymal stem cells; SCX, scleraxis.

Statistical analysis. Data are presented as the mean \pm standard deviation (SD), and analysis of variance (one-way ANOVA) was performed to compare the differences among the 5 groups with post hoc LSD test. Statistical analyses were performed using SPSS 13.0 statistics software (SPSS Inc., Chicago, IL, USA). $\mathrm{P}<0.05$ was considered to indicate statistical significance.

\section{Results}

BMP-12 induces MSCs tenocyte differentiation. SCX is predominantly expressed in tendons and is considered the most reliable phenotypic marker of the tenocytic lineage. The expression levels of SCX, collagen I and collagen III at 48 h, 5 and 7 days are shown in Fig. 3. Although BMP-12 was removed after $48 \mathrm{~h}$, the expression of SCX continued to increase until 7 days $(\mathrm{P}<0.01)$. Collagen I and collagen III show a reversal of the pattern, i.e., their expressions require persistent induction by BMP-12. The expression of collagen I is more sensitive than the expression of collagen III, with a rapid increase in collagen $\mathrm{I}$ at $48 \mathrm{~h}(\mathrm{P}<0.01)$.

Induced MSCs enhance ultimate tensile strength of linea alba incision. There was no death or hernia formation in the study. Because of the tearing of the muscles during the tensiometric test, one piece of the samples was wasted in groups A, B and D, and three pieces in group E. The comparison of the 5 groups is presented in Fig. 4. The max tension of the normal linea alba is $8.62 \pm 0.94 \mathrm{~N}$. The tension levels of groups B, C, D and E are 44, 41.8, 51.6 and $69.7 \%$ compared to group $\mathrm{A}$. The difference is not significant between groups $\mathrm{B}, \mathrm{C}$ and $\mathrm{D}(\mathrm{P}>0.05)$. Group $\mathrm{E}$ exhibits a greater increase in tension than groups $\mathrm{B}, \mathrm{C}$ and $\mathrm{D}$, with increases of 58.6, 66.9 and $35.1 \%$, respectively $(\mathrm{P}<0.05)$.

Induced MSCs enhance collagen remodeling of linea alba incision. There are 9 histological examples in Group A, B and D, 10 histological examples in Group $\mathrm{C}$ and 7 examples in Group E. Masson's histological analysis demonstrates that the tenogenic differentiation of MSCs can obviously enhance the healing of the linea alba incision (Table III), as shown in histological sections (Fig. 5). Collagen organization and amount of Group $\mathrm{E}$ is better than other three groups $(\mathrm{P}<0.05)$. Collagen organization of Group D is better than group B and $\mathrm{C}$, but worse than group $\mathrm{E}(\mathrm{P}<0.05)$. By contrast, the fibrous matrix are significantly disorderly organized in Group B and $\mathrm{C}$ (Fig. 5Ba-Bb, Fig. 5Ca-Cb).

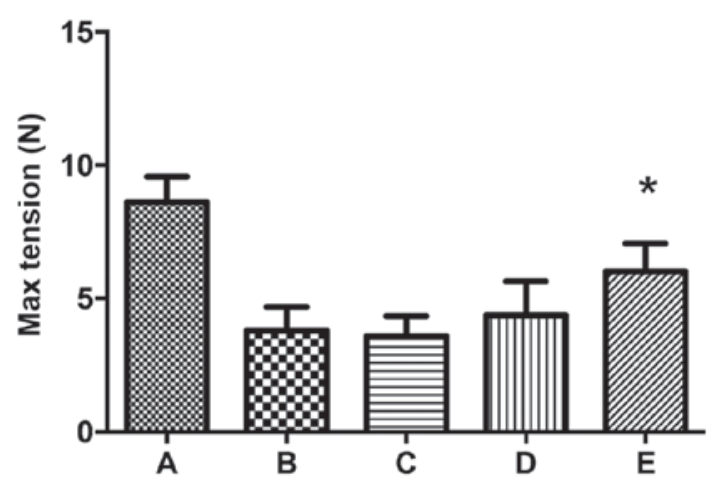

Figure 4. Induced MSCs enhance ultimate tensile strength of linea alba incision. Group E of tenogenically differentiated MSCs exhibits more increased tension than positive control group B, sham group C and native MSCs group $\mathrm{D}$, with increases of 58.6, 66.9 and $35.1 \%$, respectively $(\mathrm{P}<0.05) .{ }^{*} \mathrm{P}<0.05$ Group A did not undergo any operation and nothing was implanted. Group B only underwent operation, but did not have anything implanted. Group C underwent operation, but only sponge scaffold was implanted into the linea alba incision. Group D underwent operation and sponge scaffold with native MSCs were implanted. Group E underwent operation and sponge scaffold with tenogenically differentiated MSCs were implanted. MSCs, mesenchymal stem cells.

\section{Discussion}

In recent years, great progress has been made in the field of abdominal wall and hernia surgery, especially in terms of materials science. Bioactive patch can remodel the abdominal wall structure after implanted and inspires widespread interest of researchers. Regarding MSCs in herniorrhaphy, few studies have performed pilot explorations in this field. Gao et al (20) tried to analyze the coating ability of various meshes of MSCs, and four other articles investigated the curative effects of hernia repair with surgical mesh in combination with MSCs (21-24). In short, the use of MSCs for regeneration is a novel field in abdominal wall surgery. As Tez and Kiliç (25) indicated the stem cell origin theory of the inguinal hernia, many further works are needed on MSCs, fascia, prosthesis, heterogeneity and herniorrhaphy.

Many studies have demonstrated that exogenous BMP-12 can induce MSCs to undergo tenogenic differentiation with increasing expression of special markers. However, this effect seems time dependent and thus is more important for downstream target genes. SCX is a tendon/ligament-specific transcription factor that is required for tenogenic differentiation (26-28). Our results reveal that BMP-12 dramatically increases SCX expression when present, which is consistent 
Table III. Histological scores of microscopic examination.

\begin{tabular}{llllc}
\hline Group & $\mathrm{B}$ & $\mathrm{C}$ & $\mathrm{D}$ & $\mathrm{E}$ \\
\hline Organization & $1.6 \pm 0.7$ & $1.5 \pm 0.53$ & $2.7 \pm 0.83^{\mathrm{a}}$ & $3.5 \pm 0.98$ \\
Amount & $1.2 \pm 0.42$ & $1.6 \pm 0.7$ & $1.3 \pm 0.48$ & $2.7 \pm 0.82^{\mathrm{a}}$ \\
\hline
\end{tabular}

${ }^{\mathrm{a}} \mathrm{P}<0.05$.

A
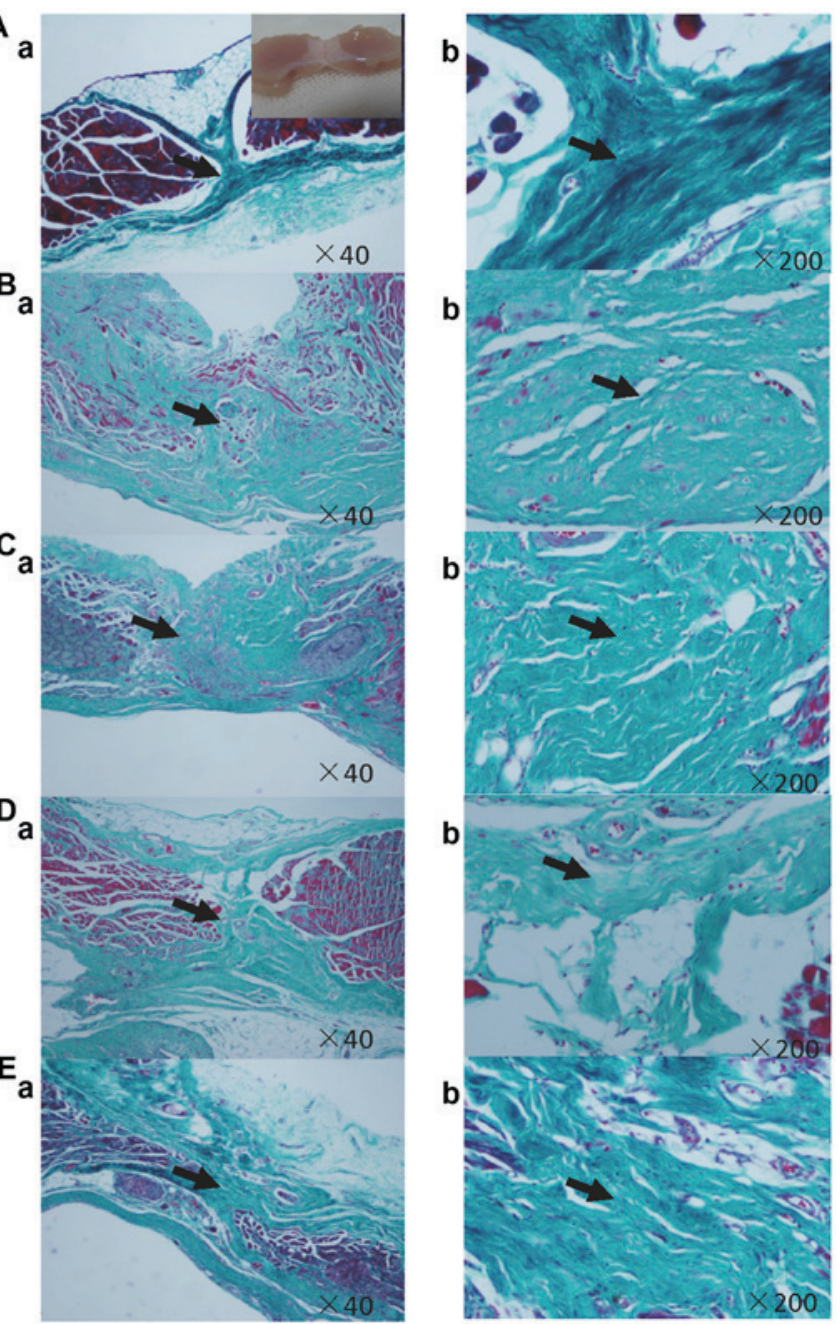

Figure 5. Masson's Trichrome staining. (A) Negative control, (B) positive control, (C) sham group, (D) native MSCs and (E) tenogenically differentiated MSCs. Group E of tenogenically differentiated MSCs demonstrates rich and well aligned collagen fibrous matrix along the transversal (tensile) axis of the incision. By contrast, the fibrous matrix in positive control group B and sham group $\mathrm{C}$ are significantly disorderly in organization. Organization and amount of collagen fibrous of native MSCs group D are better than group B and $\mathrm{C}$, but worse than group $\mathrm{E}$, which is corresponding to the semiquantitative histological analysis (Table III). Aa, Ba, Ca, Da, Ea x40; Ab, Bb, Cb, Db, Eb x200. MSCs, mesenchymal stem cells.

with other studies $(10,14)$. However, the expression was previously reported to decrease when BMP-12 was absent (14). Conversely, expression continues to increase in our study, which may be attributable to the longer inducing time. In short, gene transfection of BMP-12 into MSCs seems to be a reliable method to continually induce the tenogenic differentiation of MSCs.
We select the most two direct methods to evaluate the healing results, including tensiometric testing and histological analysis. Tensile strength corresponds to the abdominal pressure, and the ultimate result means that the healing incision can bear some violent activities, such as cough and jump. Histological analysis presents that tenogenic differentiation of MSCs in treating the linea alba incision demonstrates the relatively rich and well aligned collagen fibrous matrix along the transversal (tensile) axis of the incision. Native MSCs can only improve the organization other than the amount of the regenerating collagen fibers. Regrettably, there are some insignificances in the semiquantitative histological analysis. Measuring diameters of the regenerating collagen fibers using transmission electron microscopy may be more convincing. However, we believe the results of our study are also conclusive as that was described by Rice et al (17).

In this study, we use a collagen sponge as an implant scaffold. However, the collagen scaffold is an interference factor for exploring the curative effect of MSCs. Thus, group C transplants with only the collagen sponge served as the sham group. There are two main reasons to involve the collagen sponge in this trial. First, MSCs require continuing induction by BMP-12 to undergo tenogenic differentiation, and collagen is a better carrier than buffer or hyaluronan paste (29). At one, seven, and fourteen days, approximately 80,45 , and $8 \%$, respectively, of the initial dose of BMP-12 is retained at the implant sites. Second, a collagen sponge can provide a confined space scaffold for the MSCs. For the healing of midline laparotomy incisions, we only want to increase the production of collagen in the linea alba, and a sponge scaffold provides solidity to prevent the diffusion of MSCs.

In our study, we confirm that BMP-12 induction of tenogenic differentiation of MSCs enhances the healing of linea alba incisions.

\section{Acknowledgements}

The authors would like to thank Dr Feng Cai (Southeast University Medical School, Nanjing, China) for his support in the isolation and culture of MSCs.

\section{Funding}

The present study was supported by Southeast University Fundamental Research Fund (grant no. 2242017K40256).

\section{Availability of data and materials}

The datasets used and/or analyzed during the current study are available from the corresponding author on reasonable request.

\section{Authors' contributions}

DW isolated the MSCs, and performed tenogenic differentiation and data analysis. J-MW performed the animal experiments. Y-YT performed the tensiometric testing and histological analysis. Z-LJ designed this study, analyzed and interpreted some data, and revised the manuscript critically for important intellectual content. As the corresponding author, he also gave final approval of the version to be published. All authors read and approved the final manuscript. 


\section{Ethics approval and consent to participate}

All experiments were conducted with the approval of the Animal Care and Use Committee of Southeast University (approval no. 20140912).

\section{Patient consent for publication}

Not applicable.

\section{Competing interests}

The authors declare that they have no competing interests.

\section{References}

1. Sanders DL and Kingsnorth AN: The modern management of incisional hernias. BMJ 344: e2843, 2012.

2. Gokani SA, Elmqvist KO, El-Koubani O, Ash J, Biswas SK and Rigaudy M: A cost-utility analysis of small bite sutures versus large bite sutures in the closure of midline laparotomies in the United Kingdom National Health Service. Clinicoecon Outcomes Res 10: 105-117, 2018.

3. Franz MG: The biology of hernia formation. Surg Clin North Am 88: 1-15, 2008.

4. Axer H, Keyserlingk DG and Prescher A: Collagen fibers in linea alba and rectus sheaths I. General scheme and morphological aspects. J Surg Res 96: 127-134, 2001.

5. Axer H, von Keyserlingk DG and Prescher A: Collagen fibers in linea alba and rectus sheaths II. Variability and biomechanical aspects. J Surg Res 96: 239-245, 2001.

6. Yildiz M, Yigit O, Sünter AV, Edizer DT, Dursun N and Okcu O: Effects of intracordal estradiol and dexamethasone injection on wound healing in vocal fold injuries. J Voice pii: S0892-1997: 30557-X, 2018

7. Stoff A, Rivera AA, Sanjib Banerjee N, Moore ST, Michael Numnum T, Espinosa-de-Los-Monteros A, Richter DF, Siegal GP, Chow LT, Feldman D, et al: Promotion of incisional wound repair by human mesenchymal stem cell transplantation. Exp Dermatol 18: 362-369, 2009.

8. Heffner JJ, Holmes JW, Ferrari JP, Krontiris-Litowitz J, Marie H, Fagan DL, Perko JC and Dorion HA: Bone marrow-derived mesenchymal stromal cells and platelet-rich plasma on a collagen matrix to improve fascial healing. Hernia 16: 677-687, 2012.

9. Gelberman RH, Linderman SW, Jayaram R, Dikina AD, Sakiyama-Elbert S, Alsberg E, Thomopoulos S and Shen H: Combined administration of ASCs and BMP-12 promotes an M2 macrophage phenotype and enhances tendon healing. Clin Orthop Relat Res 475: 2318-2331, 2017.

10. Shen H, Gelberman RH, Silva MJ, Sakiyama-Elbert SE and Thomopoulos S: BMP12 induces tenogenic differentiation of adipose-derived stromal cells. PLoS One 8: e77613, 2013.

11. Lennon DP and Caplan AI: Isolation of rat marrow-derived mesenchymal stem cells. Exp Hematol 34: 1606-1607, 2006.

12. Cai F, Wu XT, Xie XH, Wang F, Hong X, Zhuang SY, Zhu L, Rui YF and Shi R: Evaluation of intervertebral disc regeneration with implantation of bone marrow mesenchymal stem cells (BMSCs) using quantitative T2 mapping: A study in rabbits. Int Orthop 39: 149-159, 2015.

13. Livak KJ and Schmittgen TD: Analysis of relative gene expression data using real-time quantitative PCR and the 2(-Delta Delta C(T)) method. Methods 25: 402-408, 2001.

14. Lee JY, Zhou Z, Taub PJ, Ramcharan M, Li Y, Akinbiyi T, Maharam ER, Leong DJ, Laudier DM, Ruike T, et al: BMP-12 treatment of adult mesenchymal stem cells in vitro augments tendon-like tissue formation and defect repair in vivo. PLoS One 6: e17531, 2011.

15. Harth KC, Broome AM, Jacobs MR, Blatnik JA, Zeinali F, Bajaksouzian S and Rosen MJ: Bacterial clearance of biologic grafts used in hernia repair: An experimental study. Surg Endosc 25: 2224-2229, 2011.
16. Tharappel JC, Ramineni SK, Reynolds D, Puleo DA and Roth JS: Doxycycline impacts hernia repair outcomes. J Surg Res 184: 699-704, 2013.

17. Rice RD, Ayubi FS, Shaub ZJ, Parker DM, Armstrong PJ and Tsai JW: Comparison of Surgisis, AlloDerm and Vicryl woven mesh grafts for abdominal wall defect repair in an animal model. Aesthetic Plast Surg 34: 290-296, 2010.

18. van Zuijlen PP, de Vries HJ, Lamme EN, Coppens JE, van Marle J, Kreis RW and Middelkoop E: Morphometry of dermal collagen orientation by Fourier analysis is superior to multi-observer assessment. J Pathol 198: 284-291, 2002.

19. Marcos-Garcés V, Harvat M, Molina Aguilar P, Ferrández Izquierdo A and Ruiz-Saurí A: Comparative measurement of collagen bundle orientation by Fourier analysis and semiquantitative evaluation: Reliability and agreement in Masson's trichrome, Picrosirius red and confocal microscopy techniques. J Microsc 267: 130-142, 2017.

20. Gao Y, Liu LJ, Blatnik JA, Krpata DM, Anderson JM, Criss CN, Posielski N and Novitsky YW: Methodology of fibroblast and mesenchymal stem cell coating of surgical meshes: A pilot analysis. J Biomed Mater Res B Appl Biomater 102: 797-805, 2014.

21. Mestak O, Matouskova E, Spurkova Z, Benkova K, Vesely P, Mestak J, Molitor M, Pombinho A and Sukop A: Mesenchymal stem cells seeded on cross-linked and noncross-linked acellular porcine dermal scaffolds for long-term full-thickness hernia repair in a small animal model. Artif Organs 38: 572-579, 2014.

22. Bogdan VG, Zafranskaya MM, Gain YM, Demidchik YE, Bagatka SS and Ivanchik GI: Modification of collagen formation by mesenchymal stem cells isolated from human adipose tissue in culture and after autotransplantation for abdominal hernia plasty. Bull Exp Biol Med 156: 152-155, 2013.

23. Majumder A, Gao Y, Sadava EE, Anderson JM and Novitsky YW: Cell-coating affects tissue integration of synthetic and biologic meshes: Comparative analysis of the onlay and underlay mesh positioning in rats. Surg Endosc 30: 4445-4453, 2016.

24. Hoganson DM, Meppelink AM, Hinkel CJ, Goldman SM, Liu XH, Nunley RM, Gaut JP and Vacanti JP: Differentiation of human bone marrow mesenchymal stem cells on decellularized extracellular matrix materials. J Biomed Mater Res A 102: 2875-2883, 2014

25. Tez M and Kiliç YA: Stem cell origin theory of the inguinal hernia. Med Hypotheses 66: 1042-1043, 2006.

26. Shukunami C, Takimoto A, Nishizaki Y, Yoshimoto Y, Tanaka S, Miura S, Watanabe H, Sakuma T, Yamamoto T, Kondoh G and Hiraki Y: Scleraxis is a transcriptional activator that regulates the expression of Tenomodulin, a marker of mature tenocytes and ligamentocytes. Sci Rep 8: 3155, 2018.

27. Le W, Cheah AE and Yao J: Ex-vivo tendon repair augmented with bone marrow derived mesenchymal stem cells stimulated with myostatin for tenogenesis. J Hand Surg Asian Pac Vol 23: 47-57, 2018

28. Docheva D, Hunziker EB, Fässler R and Brandau O: Tenomodulin is necessary for tenocyte proliferation and tendon maturation. Mol Cell Biol 25: 699-705, 2005.

29. Seeherman HJ, Archambault JM, Rodeo SA, Turner AS, Zekas L, D'Augusta D, Li XJ, Smith E and Wozney JM: rhBMP-12 accelerates healing of rotator cuff repairs in a sheep model. J Bone Joint Surg Am 90: 2206-2219, 2008.

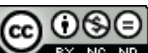

This work is licensed under a Creative Commons Attribution-NonCommercial-NoDerivatives 4.0 International (CC BY-NC-ND 4.0) License. 